\title{
LESS IS MORE
}

\section{Targeting Vascular Risk Factors in Older Adults From Polypill to Personalized Prevention}

\author{
Enrico Mossello, MD, PhD
}

For many years vascular disease prevention strategies have been focused on reducing undertreatment, often using a "one-size-fits-all" approach to increase patient adherence. The paradigm of this approach has been the proposal of a polypill targeting multiple vascular risk factors, a stanRelated articles dard treatment aimed at maximizing vascular protection. Conversely, during recent years an increasing emphasis has been placed on the issue of potential overtreatment, frequently resulting from the same treatment approach in all patients despite significant individual differences in comorbidity and life expectancy. In type 2 diabetes mellitus (T2DM), data from randomized clinical trials ${ }^{1}$ have shown uncertain or negative benefit-risk trade-offs associated with aggressive treatment of hypertension and hyperglycemia. These results, added to the well-known exclusion of elderly individuals with multiple morbidities from nearly all clinical trials, have informed recent guidelines, which now recommend more moderate targets for treatment of T2DM and hypertension in older participants, especially those considered frail or affected by important comorbidities. ${ }^{1}$

These treatment recommendations raise the possible strategy of treatment deintensification for high blood pressure and hyperglycemia in patients with values previously identified as optimal and now labeled as low. This issue was addressed by Sussman and colleagues ${ }^{2}$ in a large retrospective sample of older individuals with T2DM (mean age, 78 years) from the Veterans Health Administration actively treated for T2DM or high blood pressure. They found that treatment deintensification (ie, dosage reduction or drug withdrawal) was performed in one-quarter or less of participants with low blood pressure (systolic blood pressure $<130 \mathrm{~mm} \mathrm{Hg}$ or diastolic blood pressure $<65 \mathrm{~mm} \mathrm{Hg}$ ) or a low hemoglobin $\mathrm{A}_{1 \mathrm{c}}\left(\mathrm{HbA}_{1 \mathrm{c}}\right)$ level $(<6.5 \%)$ (to convert to a proportion of total hemoglobin, multiply by 0.01 ). Moreover, whether treatment reduction occurred was only weakly associated with a patient's blood pressure or $\mathrm{HbA}_{1 \mathrm{c}}$ level and predicted life expectancy, suggesting that physicians are generally reluctant to deintensify treatment even in conditions that make benefits of therapy limited in comparison with potential harms.

This interpretation is confirmed by a further study from the Department of Veterans Affairs in the present issue of JAMA Internal Medicine. Caverly et $\mathrm{al}^{3}$ surveyed a national sample of health care professionals providing primary care, showing that almost half of them would not worry about harms of tight glycometabolic control obtained with an insulin secretagogue in an older patient at high risk for hypoglycemia. This approach to therapy was largely explained by the concern of making the patient's $\mathrm{HbA}_{1 \mathrm{c}}$ level fall out of Department of Veterans Affairs performance measures, which, as the authors point out, have never targeted values less than $7.0 \%$. Nearly one-quarter of the health care professionals interviewed would even be concerned about malpractice liability risk with deintensification of hypoglycemic medications.

In keeping with these data, Sussman and colleagues ${ }^{2}$ call for a change in guidelines, quality measures, and clinical performance management that should include recommendations and incentives to avoid overtreatment. This statement is also consistent with a recent analysis of outpatient and emergency department performance measures in the United States that shows a lack of measures addressing overuse, especially regarding treatments. ${ }^{4}$ Yet, some caveats should be raised. Although studies of more aggressive control of risk factors have generally failed to show a reduction of cardiovascular events in T2DM, few data exist regarding the prognostic effect of deintensification, especially in patients with good treatment tolerance. Conversely, more stringent targets are still recommended, although with a low level of evidence, for younger patients with a low risk for adverse events. ${ }^{1}$ In addition, incentives for treatment withdrawal might carry a risk of avoiding potentially useful preventive strategies, especially in the present time of resource constraints. Less is not always more, and we should not risk eliminating the benefits of therapy while attempting to lower the risk.

A proposed stratification of antihypertensive and antidiabetic treatment strategies according to patient vulnerability is described in the Figure, including issues that should be further clarified and possible solutions. A first step is represented by the need for simple but clear indicators of vulnerability, which may suggest the need for less-stringent control of risk factors. According to the limited evidence available, a less-stringent $\mathrm{HbA}_{1 \mathrm{c}}$ target level in the treatment of diabetes might be associated with a better prognosis in patients with T2DM with overt disability, ${ }^{5}$ older age, and more severe comorbidity, especially if they are receiving insulin. ${ }^{6}$ Moreover, in observational studies of individuals without diabetes, antihypertensive treatment leading to lower blood pressure values was associated with greater mortality risk in elderly nursing home residents ${ }^{7}$ and a negative effect in participants with cognitive impairment ${ }^{8}$ and slow gait speed. ${ }^{9}$ These observations should be replicated in large observational studies. As a further step, appropriate clinical trials on deintensification of vascular risk factors treatment in specifically identified vulnerable groups should be designed to assess the effects of less aggressive control and identify appropriate targets for intervention. 
Figure. Proposed Stratification of Antihypertensive and Antidiabetic Treatment in Type 2 Diabetes Mellitus According to Patient Vulnerability

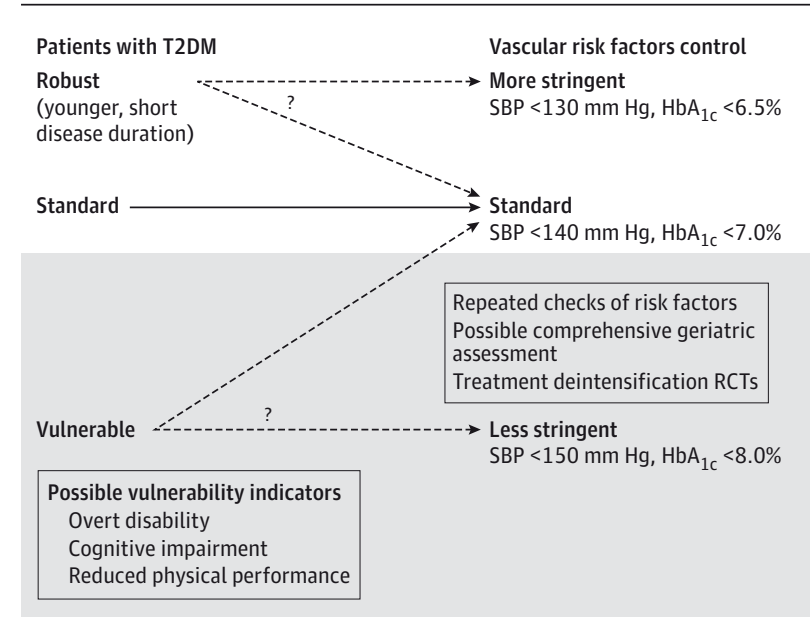

Solid line with arrowhead indicates standard vascular risk control strategy. Dashed lines with arrowheads indicate possible vascular risk control strategies for robust and vulnerable patients, with question marks pointing out the need for a choice. Gray box presents the proposed approach for vulnerable individuals. $\mathrm{HbA}_{1 \mathrm{c}}$ indicates hemoglobin $\mathrm{A}_{1 \mathrm{c} i} \mathrm{RCTs}$, randomized clinical trials; SBP, systolic blood pressure; and T2DM, type 2 diabetes mellitus. To convert $\mathrm{HbA}_{1 \mathrm{c}}$ to a proportion of total hemoglobin, multiply by 0.01 .
In waiting for such evidence, it is reasonable to use available vulnerability indicators as designators for a more cautious approach to vascular risk factor prevention. In the study by Sussman et al, ${ }^{2}$ patients who had the lower frequency of blood pressure or $\mathrm{HbA}_{1 \mathrm{c}}$ monitoring also had the lowest rate of treatment discontinuation. This finding suggests that low values for these measures is not perceived as a potential problem and, conversely, that more strict control, at least initially, will probably be needed for deintensification of treatment. In more complex, selected cases, vulnerable patients might be referred for a comprehensive geriatric assessment, including risk for falls, hypoglycemia, treatment nonadherence, and comorbidity, to appropriately balance risks and benefits of preventive treatment. This kind of approach would increase the number of clinical assessments for vulnerable individuals along with a possible increase in costs, but this increase could be counterbalanced by savings from a decreased number of prescriptions and the expected reduction of adverse events. Therefore, clinical performance measures coupling treatment deintensification with appropriate clinical assessments and monitoring seem reasonable to safely discontinue unnecessary and potentially harmful treatments while retaining the benefits of vascular prevention. This approach will be key to designing effective personalized prevention strategies for older vulnerable subjects.

\section{ARTICLE INFORMATION}

Author Affiliation: Division of Geriatric Medicine and Cardiology, Department of Experimental and Clinical Medicine, University of Florence and Careggi Teaching Hospital, Florence, Italy.

Corresponding Author: Enrico Mossello, MD, PhD, Division of Geriatric Medicine and Cardiology, Department of Experimental and Clinical Medicine, University of Florence and Careggi Teaching Hospital, Viale G. Pieraccini 6, Florence 50141, Italy (enrico.mossello@unifi.it).

Published Online: October 26, 2015. doi:10.1001/jamainternmed.2015.5941.

Conflict of Interest Disclosures: None reported.

\section{REFERENCES}

1. Fox CS, Golden SH, Anderson C, et al; American Heart Association Diabetes Committee of the Council on Lifestyle and Cardiometabolic Health, Council on Clinical Cardiology, Council on Cardiovascular and Stroke Nursing, Council on Cardiovascular Surgery and Anesthesia, Council on Quality of Care and Outcomes Research, and the American Diabetes Association. Update on prevention of cardiovascular disease in adults with type 2 diabetes mellitus in light of recent evidence: a scientific statement from the American Heart Association and the American Diabetes Association. Circulation. 2015;132(8):691-718

2. Sussman JB, Kerr EA, Saini SD, et al. Rates of deintensification of blood pressure and glycemic medication treatment based on levels of control and life expectancy in older patients with diabetes mellitus [published online October 26, 2015]. JAMA Intern Med. doi:10.1001/jamainternmed.2015.5110.

3. Caverly TJ, Fagerlin A, Zikmund-Fisher BJ, et al. Appropriate prescribing for patients with diabetes at high risk for hypoglycemia: national survey of Veterans Affairs health care professionals [published online October 26, 2015]. JAMA Intern Med. doi:10.1001/jamainternmed.2015.5950.

4. Newton EH, Zazzera EA, Van Moorsel G, Sirovich BE. Undermeasuring overuse-an examination of national clinical performance measures [published online August 10, 2015]. JAMA Intern Med. doi:10.1001/jamainternmed.2015.4025.

5. Yau CK, Eng C, Cenzer IS, Boscardin WJ, Rice-Trumble K, Lee SJ. Glycosylated hemoglobin and functional decline in community-dwelling nursing home-eligible elderly adults with diabetes mellitus. J Am Geriatr Soc. 2012;60(7):1215-1221.

6. Monami M, Vitale V, Lamanna C, et al. $\mathrm{HbA}_{1 \mathrm{c}}$ levels and all-cause mortality in type 2 diabetic patients: epidemiological evidence of the need for personalised therapeutic targets. Nutr Metab Cardiovasc Dis. 2013;23(4):300-306.

7. Benetos A, Labat C, Rossignol P, et al. Treatment with multiple blood pressure medications, achieved blood pressure, and mortality in older nursing home residents: the PARTAGE study. JAMA Intern Med. 2015;175(6):989-995.

8. Mossello E, Pieraccioli M, Nesti N, et al. Effects of low blood pressure in cognitively impaired elderly patients treated with antihypertensive drugs. JAMA Intern Med. 2015;175(4):578-585.

9. Odden MC, Peralta CA, Haan MN, Covinsky KE. Rethinking the association of high blood pressure with mortality in elderly adults: the impact of frailty. Arch Intern Med. 2012;172(15):1162-1168. 